\title{
LAS FUERZAS ARMADAS Y LOS NUEVOS PARADIGMAS DE LA SEGURIDAD INTERNA
}

\author{
Mayo de I. David Eliseo Pico Medina ${ }^{1}$ \\ Mayo de Int. Christian Gabriel Moya Arias ${ }^{2}$ \\ RESUMEN
}

Haciendo un estudio sobre los cambios en la seguridad interna se ha propuesto este trabajo enfocar el tema en tres aspectos fundamentales que permite un análisis del nuevo paradigma militar como complemento afín. Partiendo del significado etimológico, su transformación al igual que las circunstancias históricas que han provocado su nueva delimitación, se realiza una exploración de la situación latinoamericana y la participación de las fuerzas armadas en la seguridad interna, de igual manera explicando el cambio del paradigma de seguridad dados los acontecimientos que han inducido a las evoluciones paradigmáticas. Para complementar lo anterior, se revisa el contexto de Ecuador, al igual que los otros países de la región viene enfrentando problemas en su seguridad, presentándose como tema controversial la intervención de las FF.AA. para poder enfrentar las amenazas multidimensionales del nuevo siglo. Se finaliza el trabajo entablando las conclusiones respectivas sobre la conjunción del nuevo paradigma militar y el de la seguridad interna y la obligatoriedad de la participación de un soldado profesional y tecnificado.

Palabras clave. - Paradigma de seguridad, Paradigma militar, Seguridad interna, Amenazas asimétricas, Seguridad integral.

\begin{abstract}
Making a study on the changes in internal security, this work has been proposed to focus on three fundamental aspects that allow an analysis of the new military paradigm as a related complement. Starting from the etymological meaning, its transformation, as well as the historical circumstances that have caused its new delimitation, an exploration of the Latin American situation and the participation of the armed forces in internal security is carried out, in the same way explaining the change in the security paradigm. given the events that have induced paradigmatic developments. To complement the above, the context of Ecuador is reviewed, as the other countries of the region have been facing security problems, presenting the intervention of the FF.AA. as a controversial issue. in order to face the multidimensional threats of the new century. The work is completed by introducing the respective conclusions on the conjunction of the new military paradigm and that of intenal security and the mandatory participation of a professional and technified soldier.
\end{abstract}

Keywords. - Security Paradigm, Military Paradigm, Internal Security, Asymmetric Threats, Integral Security.

\footnotetext{
1 piquete93@gmail.com

ACADEMIA DE GUERRA

2 tigremoya1975@hotmail.com

ACADEMIA DE GUERRA
} 


\section{Introducción}

Los cambios de factores que alteran la seguridad de un país han provocado transformaciones en los paradigmas enfocados a la seguridad integral y como complemento afín a la seguridad interna el paradigma militar. El análisis de estos dos conlleva a reflexionar sobre las amenazas asimétricas, multidimensionales, no tradicionales denominadas así al crimen organizado, narcotráfico y terrorismo con todas sus consecuencias que estás transfieren

Estas conjeturas nos ha llevado a este trabajado titulado "Las Fuerzas Armadas y los nuevos paradigmas de la seguridad interna" que tiene como propósito analizar la limitación que abarca este concepto, relacionado con el paradigma militar y el de la seguridad interna al ser tratados de manera tradicional y conservadora. Este estudio se dividió en tres partes principales: la primera considera la conceptualización del tema integrando el aspecto militar y la seguridad interna. La segunda estudia el ambiente latinoamericano, resaltando los acontecimientos del último lustro; y, el tercero se centra en la situación de Ecuador frente a la seguridad interna exponiendo los sucesos de finales de 2019, enfocándose además en este epígrafe la relación de los acontecimientos y la necesidad de aceptar los cambios de los paradigmas descritos.

Para realizar esta investigación nos hemos planteado las siguientes hipótesis: Las Fuerzas Armadas necesitan ampliar su doctrina para enfrentar las amenazas multidimensionales; $\mathrm{y}$, la segunda hipótesis propuesta dice que los nuevos paradigmas militar y de la seguridad interna están relacionados entre sí.

Aplicando una metodología investigativa, analítica, descriptiva se pudo llegar a narrar los diferentes casos que se exponen, para finalmente a través de las conclusiones demostrar las hipótesis trazadas.

\section{Primera parte}

\section{Definición de paradigma}

En primera instancia se hace imperante explicar el origen del término "paradigma" que deriva de la lengua griega paradiegma traduciéndose como "ejemplo que sirve de norma". Esta definición se complementa con sinónimos de la palabra paradigma como muestra, prototipo, arquetipo, ideal, precedente, regla, entre otras similitudes (Acosta, 2009)

Joel Barker en su libro "Paradigma" dice que un paradigma es un conjunto de reglas y disposiciones (escritas o no) que hace dos cosas: 1) establece o define límites, y 2) indica cómo comportarse dentro de los límites para tener éxito.

Adam Smith (1723-1790) conocido como el fundador del capitalismo declaraba que "El paradigma nos explica el mundo y nos ayuda a predecir su comportamiento". (Citado en Capponi, 2019)

A pesar de los muchos conceptos creados alrededor de la definición de paradigma, la más acertada y acogida por la ciencia es la promulgada por el filósofo Thomas Kuhn manifestando que "Paradigma son las realizaciones científicas universalmente reconocidas que, durante cierto tiempo, proporcionan modelos de problemas y soluciones a una comunidad científica" (Kuhn, 1971, pág. 13) Por último, Kuhn (1971) considera revolución científica a "la transición de un paradigma en crisis a otro nuevo del que pueda surgir una nueva tradición de ciencia normal" (pág. 36). En su explicación Kuhn afirma que el cambio de paradigma surge luego de comprobarse la aparición de nuevas interpretaciones alternativas que han emergido por diferentes factores que lo rodean.

\section{Nuevos paradigmas}

Los nuevos paradigma están relacionados con la inconformidad de la relación entre los conocimientos adquiridos y la actualidad, considerados nuevos y revolucionarios dados los cambios extremos que la humanidad está viviendo, volviéndose su implementación de forma radical, ajustándose a los nuevos conceptos y reinterpretando con un paradigma emergente.

Para desarrollar el nuevo concepto de paradigma de seguridad es importante señalar el concepto tradicional de seguridad afianzada en la definición de seguridad nacional establecida por George Kennan en 1948 y dice que "es la capacidad continua de un país para proseguir el desarrollo de su vida interna sin interferencia seria, o amenaza de interferencia de potencias extranjeras" (Centro Internacional de Toledo por la Paz CITpax, 2009, pág. 11)

Los impactos sufridos a nivel mundial y en la última década específicamente en la región latinoamericana han hecho tambalear a los paradigmas de seguridad existentes. El fenómeno de la inseguridad ha generado nuevas propuestas para desarrollar un concepto moderno, integral y sostenible de seguridad, en consecuencia, Margarita Magaña en su artículo "El otro paradigma de la seguridad" dice:

"En el informe presentado en mayo de 2003, la Comisión de Seguridad Humana ya alertaba de la necesidad urgente de que la comunidad internacional estableciera un nuevo paradigma de seguridad, ya que el paradigma tradicional de la seguridad -que data del siglo XVII-, en el cual el Estado monopoliza los derechos y medios para proteger a sus ciudadanos en el orden y la paz, había sido superado por los nuevos desafíos del siglo XXI".

(Magaña, 2009, pág. 128) 
Esto se demuestra puesto que la respuesta de los Estados a la seguridad se ha venido deteriorando y haciéndose más ineficiente, radicando la crisis del paradigma estatocéntrico (llamado también realista) de la seguridad en la evolución de los nuevos peligros y amenazas, como: "terrorismo, redes internacionales de narcotráfico, delincuencia organizada, degradación del medio ambiente, pandemias como el VIH-SIDA, crisis financieras mundiales, extrema pobreza, flujos migratorios no controlados, etc.” (Magaña, 2009, pág. 128).

Entonces se puede afirmar que es necesario ampliar los límites del paradigma de seguridad, estableciendo uno "innovador y propositivo" (Jarrín, 2011), disgregando la posibilidad del contexto político y el abuso mediático. Por lo tanto, basarse en conceptos expuestos en la década pasada es totalmente decadente y desactualizado. Así como la tecnología avanza, la inseguridad se dilata, crece y configura nuevos actores.

Si asociamos al bien común con seguridad adaptada a la fuerza de tipo militar que un Estado esté preparado para ejercer se debería aceptar un nuevo, amplio y comprensivo concepto de seguridad, definiendo el paradigma de la verdadera seguridad, explicando que esta no se puede lograr por una carrera armamentista dependiendo solo del balance militar, sino de la cooperación global para asegurar un ambiente sustentable y sostenida prosperidad (Brandt, 1980)

\section{Segunda parte}

\section{El paradigma de la seguridad}

Han pasado 70 años del planteamiento de paradigma de seguridad expuesto por el ya citado George Kennan, restringido entre Estado y Nación, excluyendo a la gente común y la exigencia de mantener seguridad en su cotidianidad. La globalización y los diferentes factores que le conducen han generado una difusión de conflictos que surgen dentro de cada país y se extienden, alcanzado regiones como es el caso de Latinoamérica, donde los países integrantes están enfrentando conflictos que afectan su seguridad interna.

La fuente de estas conflagraciones en la región es el tráfico de drogas, dando paso al crimen organizado. Para Alexis Tocqueville describe al crimen en Latinoamérica como un fenómeno complejo y pluricausal, que tiene facetas múltiples que van desde la delincuencia marginal del pequeño hurto, pasando por los asaltos perpetrados por bandas armadas secuestros a empresarios y robo a entidades bancarias hasta alcanzar los más graves como es el crimen organizado internacional (Álvarez, Garzón, Tellería, \& Paz, 2012).

Adicionalmente a esto, se ha observado que los últimos hechos suscitados en Latinoamérica se suman otros con encadenamiento social que están afectando internamente y de manera más directa que las mismas amenazas multidimensionales ya expuestas. "Los mo- vimientos sociales suelen asociarse a grandes palabras, tales como rebeldía, lucha, resistencia, desobediencia, insurrección, protesta" (Revilla, 2010, pág. 54), acogiéndose a un repertorio de violencia difícil de distinguir entre conflicto armado, terrorismo, lucha armada o guerrilla, aprovechándose de esta situación la delincuencia común y hasta la delincuencia organizada.

Según lo expuesto la activa participación de las fuerzas armadas en escenarios de seguridad interna está trayendo consigo problemas de diversa naturaleza, entre ellos, el interrogante acerca de su intervención en asuntos democráticos, la verdadera subordinación al poder civil, la vulnerabilidad de los derechos ciudadanos y el rol constitucional de la fuerza pública y el apoyo de las Fuerzas Armadas.

En comparación con años anteriores donde las FF.AA. vienen desarrollando actividades direccionadas a la seguridad externa, siempre preparadas para la defensa en un ambiente operacional de guerra regular o irregular, no obstante, siempre estuvieron brindado su apoyo a las acciones del Estado cumpliendo con la seguridad de sectores estratégicos, seguridad ciudadana, gestión de riesgos, seguridad y control marítimo, contra el narcotráfico, crimen organizado y terrorismo, apoyo al orden público, respuesta ante las crisis, control y contrabando de combustibles, etc., trabajo que lo vienen realizando sobre todo en las fronteras, desarrollando operaciones subsidiarias y coordinadas con la Policía Nacional, la Secretaría Nacional de Gestión de Riesgos, organismos seccionales y otros. (Fuerzas Armadas del Ecuador, 2014).

Pero si se habla de un nuevo paradigma de seguridad este al igual que su concepto debe extenderse hacia enfoques multidimensionales afectados por factores políticos, militares, económicos, sociales y ambientales (Buzan, 1991), claramente expuesto en la concepción de seguridad integral concebida a través de la integración del Ser humano, la Naturaleza y El Estado, interrelacionada con la Defensa y Relaciones Internacionales, Seguridad ciudadana y Justicia, Gestión de Riesgos y Ambiente, Soberanía Tecnológica y Ciencia e Inteligencia Estratégica (Medina, 2015). Adicionalmente se debe incluir todas las dimensiones necesarias de la seguridad global y asociada a la protección de intereses universales, sin declinar ni el desarrollo del Estado ni del ser humano.

\section{El paradigma de la seguridad en Latinoamérica}

Siguiendo con el análisis del tema planteado es indispensable analizar la situación en América Latina donde formas de violencia subversiva y contrainsurgente se han hecho permanentes en la región. Estas ya no se puede direccionar solo a Colombia, las amenazas se han generalizado en toda la zona, adquiriendo características de terrorismo. 
El acelerado aumento de conflictos internos engendrado por la creciente aceleración del narcotráfico, delincuencia, organizaciones delictivas y otras amenazas con las que los Estados Latinos no han podido dar soluciones, peor aún han aumentado al punto de confrontarse con ejércitos delictivos cuyos únicos contrincantes son las Fuerzas Armadas debido a sus capacidades operativas estratégicas y tecnológicas que les hacen aptos para detener esta fuerza criminal que avanza sin poder ser eliminada.

Esto ha provocado que los países latinos recurran a la intervención de sus ejércitos que se ha visto involucrado en conflictos internos para dar soporte a la seguridad ciudadana. Adicionalmente a esto, los movimientos sociales como focos de intervención en los acontecimientos acaecidos durante el año pasado en Latinoamérica han demostrado la inconformidad de la población con el Estado.

Razonar el por qué de los conflictos internos en Latinoamérica se podría citar a Clausewitz al sostener que la guerra es la política bajo otra forma, la violencia (Borrero, 2003). En este concepto se integra sobre todo el caso colombiano, cuyo conflicto se pretende resolver por cualquier vía, ya sea con una victoria militar por cualquiera de las partes involucradas. Así los insurgentes, defendiendo su propuesta socialista enfrentados con el Estado y su propuesta democrática.

\section{México}

Son muchos los detonantes por los que Latinoamérica ha presentado un cuadro de discordia entre la sociedad y sus Estados correspondientes. Haciendo un recuento cronológico de lo sucedido en 2019 y empezando por México, el 17 de octubre el gobierno con el apoyo de militares se enfrenta a los narcotraficantes para lograr la captura del hijo del Chapo Guzmán, objetivo que no se pudo cumplir debido a la violencia que desataron los narcotraficantes pertenecientes al Cartel de Sinaloa. Ante esto el gobierno de Andrés Manuel López Obrador tuvo que ceder ante las actuaciones agresivas y violencia generalizada en la ciudad de Culiacán.

En México los problemas de narcotráfico vienen agudizándose, desembocando violencia criminal, inseguridad pública, desaparición forzada de personas. El caso mexicano se complementa con un vacío de poder, corrupción en altas esferas gubernamentales, abandono de justicia. Por tal razón el Estado Mexicano no ha podido controlar la seguridad nacional acudiendo a las FF.AA. para realizar la tarea de seguridad pública bajo el riesgo de provocar una fuerza excesiva involucrando a los derechos humanos.

Sobre el debate de "Las Fuerzas Armadas en las Calles", el autor Portillo (2011) opina que "se debe reconfigurar la doctrina militar para así incorporar una nueva base conceptual, normativa, institucional, estratégica, logística y operativa que las habilite en las coordenadas del uso racional de la fuerza" (pág. 7)

\section{Venezuela}

El caso de Venezuela envuelta en una crisis total, económica, social, energética, humanitaria, migratoria, política, con un gobierno cubierto por las FF.AA. en un papel mal interpretado de seguridad nacional, 2019 fue un año extremo para el país Bolivariano, convertido en un Estado mafioso, gobierno y oposición tiran de una cuerda llamada pueblo, auspiciados cada uno por potencias externas que quieren demostrar su protagonismo.

El problema venezolano ha trascendido de político, geopolítico y energético a regional e internacional por la intervención de EE.UU. proclamando una lucha antiimperialista de alcance regional y extrarregional. La situación se ha desbordado al punto que ha causado disgregación en Latinoamérica, con profundas divisiones al punto que los países latinos no ha podido hacer frente a un Maduro fortalecido por la misma división opositora.

El pueblo por su parte inmerso en violencia dentro de una crisis económica han sido los catalizadores para una migración masiva de aproximadamente 5 millones de venezolanos a abandonar su país considerado el mayor poseedor de reservas petrolíferas.

Junto con personas que huyen de una situación insalvable, se insertan delincuentes menores, pero la situación atrae a grupos mayores que amenazan la seguridad de los países vecinos. El éxodo venezolano es un aporte directo a la inseguridad que se ha generado en Colombia y Ecuador.

Las Fuerzas Armadas venezolanas por su parte son el soporte de Nicolás Maduro y esto es lo que le ha asegurado su permanencia en el poder. Mientras que Juan Guaidó, a pesar de tener un gran apoyo internacional y considerarse su elección como presidente constitucional, no ha podido conseguir su apoyo.

Cabe recalcar que actualmente el resquebrajamiento de las FF.AA. es evidente. Los de la alta cúpula son los privilegiados, mientras que su subalternos están viviendo en condiciones iguales que el pueblo en general, pudiendo ser este un detonante para un levantamiento proveniente de los oficiales más jóvenes, dañando la estructura militar de dominio que ha mantenido hasta el momento, siendo esta la mejor posibilidad para un cambio militar y una solución a la situación venezolana.

\section{Ecuador}

Los primeros días de octubre, Ecuador enfrenta una crisis donde intervienen ya no solo movimientos sociales que solicitan se derogue el decreto que elimina- 
ba el subsidio a la gasolina, se puede apreciar grupos violentos que su enfoque principal es la desestabilización del gobierno. La intervención de las FF.AA. en apoyo a la Policía demuestra la falta de capacidades operacionales causando un deterioro a la reputación de la institución.

\section{Chile}

Chile a continuación de Ecuador emulsiona una crisis social con el descontento político que abarca los últimos treinta años desde su retorno a la democracia. $\mathrm{Al}$ igual que los otros países, la actuación de las FF.AA. es analizada y fuertemente criticada inclusive por organismos internacionales encargados de la defensa de los Derechos Humanos.

Al respecto de emplear al personal militar en tareas que no son apropiadas a su naturaleza Oliva (2013) opina que si las Fuerzas Armadas son utilizadas para preservar la seguridad interior, significa que la autoridad civil, por cualquier causa, ha sido anulada, superada, reducida o corrompida (pág. 57). A pesar de lo citado, la consecuencia de la identificación de estas amenazas asimétricas o emergentes transnacionales, el Estado se ha visto en la obligación del empleo de las FF.AA. como apoyo para salvaguardar la seguridad interna, por lo que se han dispuesto marcos legales para limitar su intervención.

\section{Bolivia}

En Bolivia un intento de fraude adquiere una violencia generalizada en el país cuyo objetivo fue alcanzado al lograr la dimisión del presidente Evo Morales. Las FF.AA. tuvieron su intervención tanto en la defensa de la ciudadanía como en conseguir la renuncia del presidente.

La actuación de las Fuerzas Armadas Bolivianas crearon criterios controversiales. Los que pretendían defender su acción como defensores de la democracia y los que vieron como otro golpe de Estado recordando un pasado que no es favorable a las FF.AA.

\section{Colombia}

Colombia no es la excepción, aquí se han recrudecido los ataques provocados por la guerrilla y disidentes de las Farc enrolados con el narcotráfico, causando el retorno de la violencia ya anunciada por sus actores. Pero la situación en este país ya no solo es direccionada a la guerrilla y narcotráfico, el paro del 21 de noviembre demostró problemas sociales de inconformidad con el gobierno de Iván Duque. EL gobierno militarizó parte del país pese a, las protestas continuaron al igual de lo sucedido en Ecuador y Chile en días anteriores, la tónica de los enfrentamientos fue muy similar. De hecho, se pudo confirmar a grupos delincuenciales como participantes directos en actos de violencia extrema
Otros casos como el de Perú, Argentina, Uruguay, Honduras y Nicaragua enmarcados en crisis entrelazadas con la política y la economía, pero al final desencadenan inseguridad ciudadana al interior de cada país, protagonizada por la delincuencia organizada que a criterio de Bellota (2017) "cuando no hay seguridad humana ... hay un subsidio a la delincuencia organizada...es una forma alternativa de gobernabilidad" (Bellota, 2016, pág. 165)

Este panorama mantiene una tendencia generalizada en la región latinoamericana, se puede observar coyunturas de agudización de los niveles de conflictividad social por períodos y países, "Por ende, se exigen nuevos marcos doctrinarios y operativos ante el espacio difuso y modos fragmentados de actuación de las nuevas amenazas y actores transnacionales. Uno de tal modo constituye el apoyo militar a la seguridad interna y a la ciudadana". (Grupo de Trabajo en Seguridad Regional (GTSR), 2013, pág. 2)

\section{Tercera parte}

\section{Situación de Ecuador y los nuevos paradigmas: paradigma de la seguridad}

La Política de la Defensa Nacional se empieza a promulgar a través de los llamados Libros Blancos. En Ecuador el primer Libro Blanco de la Defensa Nacional se publicó en 2002. Luego de los imprevistos acontecimientos históricos desde finales de los 80 hasta comienzo de los 90 como: la caída del muro de Berlín, la reunificación de Alemania, el desmembramiento de la Unión Soviética, son los principales sucesos que eliminaron el orden bipolar mantenido hasta ese momento por parte de los dos mundos formados hasta ese entonces por las dos potencias.

Estos hechos descritos son la base para hablar de nuevos paradigmas, así se enuncia en este documento de 2002 explicando que se ha establecido "una paradoja: los sistemas y conceptos de seguridad y defensa de los países responden aún en cierta medida a un escenario que desapareció y todavía no se ha terminado de adaptar a las necesidades de los nuevos escenarios vigentes" (Red de Seguridad y Defensa de América Latina, 2002). En este Libro Blanco se describen las nuevas amenazas a la seguridad lo que exige modificar las agendas de Seguridad y Defensa para hacerles frente.

Para el 2006 se pública un nuevo Libro Blanco cuya concepción de la seguridad nacional es multidimensional y establece como prioridad la seguridad humana, diferenciando entre seguridad y defensa. Esta ultima responde a los "objetivos de la seguridad nacional, para el mantenimiento de la soberanía y protección de la población, las fronteras, los recursos naturales contra cualquier tipo de agresión" (Ministerio de Defensa Nacional, 2006). 
En La política de la Defensa Nacional del Ecuador "Libro Blanco" de 2018 se expone que en el actual escenario estratégico, una separación rígida entre los campos interno y externo no corresponde a un escenario con amenazas y riesgos comunes, que determinan la necesidad de adopción de políticas concertadas y con responsabilidades claras y bien definidas para las Fuerzas Armadas. (Ministerio de Defensa, 2018)

Sobre lo expuesto el ministro de Defensa Oswaldo Jarrín dice:

"las FF.AA. están preparadas para responder en situaciones de crisis y está claro que tienen el monopolio exclusivo del uso de la fuerza junto a la Policía Nacional. Sin embargo, es necesaria la aprobación de las leyes de seguridad y militares enviadas a la Asamblea desde el año pasado".

(El Universo, 2019)

Por su parte, el Plan de Seguridad Integral (2011) sostiene que "las Fuerzas Armadas asumen nuevos roles y tareas relacionadas con la seguridad con enfoque integral para prevenir y enfrentar las nuevas amenazas, mediante acciones concretas en apoyo a la Seguridad Interna y Orden Público" (Ministerio de Coordinación de Seguridad, 2011)

\section{Paradigma militar}

Se han identificado tres épocas para definir los paradigmas militares. El primer paradigma ubicado en la época agrícola cuyos pobladores combatían por los recursos naturales; el segundo paradigma en congruencia con la Revolución Industrial caracterizado en el heroísmo, guerras ganadas o perdidas, el caso concreto y fin de este es la Segunda Guerra Mundial; y, el tercero y actual se inicia en la Revolución de la Información que generó una sociedad posindustrial, desmasificada y descentralizada por lo tanto concibe un nuevo paradigma que sitúa al militar en el apoyo, el humanitarismo, auxilio a la población refugiada, el heroísmo se practica para socorrer al más débil no para ganar trincheras. Pero ¿qué tan efectivo es este nuevo paradigma frente al crimen organizado, la guerrilla, el terrorismo y el narcotráfico?

Relacionando lo expuesto, se puede determinar que así como se busca plantear un nuevo paradigma de la seguridad que involucre todo lo que a este concepto atañe, ampliando sus límites al enfrentamiento de amenazas asimétricas, multidimensionales y no tradicionales, con sus riesgos y peligros, el paradigma de la doctrina militar presente también necesita cambios. Este cambio de paradigma militar debe estar involucrado en el nuevo paradigma de la seguridad ampliando su participación en la seguridad interna de una nación, para poder hacer frente a "las nuevas guerras o guerras modernas" (Algora, 2012) con nuevos escenarios y actores.

Sin embargo, autores como Gómez (2012) discrepan en la construcción de un nuevo paradigma militar, para la especialista diplomática en seguridad, el paradigma militar ha sufrido una transformación, una adaptación a las nuevas tecnologías, así como la capacidad para enfrentarse a las amenazas asimétricas, el uso de la tecnología y los medios de comunicación son algunos de los factores que han transformado al profesional militar.

Es importante señalar que la actuación de las Fuerzas Armadas hoy en día no es entre Estados sino entre ciudadanos de un mismo país enfrentados entre sí, con un factor común que es la excesiva violencia. La participación de las FF.AA. como un tercer elemento es parte del mismo grupo, sin poder determinar, en algún momento, a quién defender por la confusión en el instante del conflicto, esto provoca el cambio de paradigma militar.

Todo lo investigado determina que se marcan diferencias entre el nuevo y antiguo paradigma, para el primero no hay un triunfo militar como lo era para el antiguo paradigma, el objetivo es alcanzar una situación final deseable sin actitudes heroicas. El militar de hoy se va especializando y adaptándose a las situaciones de un enemigo cambiante, así como irregular es el escenario, la tecnología y las armas con las que puede enfrentar las amenazas asimétricas. Es decir, la profesionalización de los ejércitos es el factor primordial, terminando con el paradigma de "ciudadano-soldado", adaptándose al mundo posmoderno y asimilando los valores civiles y militares (Colom, 2014).

Estas amenazas están identificadas como inferencias de la globalización y factor primordial para su proliferación, todo esto motivado por la inestabilidad de los Estados y su incapacidad para el control adecuado de la seguridad. Las fronteras se han difuminado creando amplias zonas de guerra, terrorismo y crimen, causando desplazamientos con sus graves consecuencias sociales.

Estos son los escenarios donde el militar actual debe actuar y acoplarse, su doctrina de guerra debe cambiar con una doctrina humanitaria para unos y una doctrina de defensa para otros. Por tal razón, este nuevo paradigma debe acaparar todas las circunstancias y características que encierra al profesional militar, en búsqueda de los conceptos que acojan todas las particularidades para que este cambio paradigmático de la doctrina militar sea completo y concreto.

En este punto entra otro término que acoge al paradigma militar ya definido por algunos autores como "paradigma militar posmoderno" con una convergencia hacia la Revolución en los Asuntos Militares que estudia y abarca los aspectos estratégicos para la integración de nuevas tecnologías tácticas y de formación militar. Este concepto es introducido por Andrew W. Marsharll en 1980 considerado el padre de la RMA, que involucra a la innovación y la tecnificación militares. Se incluyen además los ejércitos posmodernos, la profesionalización de la milicia caracterizada por el fin 
del modelo de ciudadano-soldado (Colom, 2014). Estos conceptos obligan a cambios sustanciales en todos los niveles de las Fuerzas Armadas.

Estos nuevos criterios no han sido ajenos ni equívocos al caso del Ecuador, se podría decir que estos se encuentran en un proceso de cambio progresivo de un tipo institucional con inclusión ocupacional, planteado desde un punto de vista estratégico por el Ministro de Defensa Oswaldo Jarrín ante la Asamblea Nacional, donde pone en consideración una defensa colaborativa con la participación de todas las instituciones del estado y el apoyo de países que buscan eliminar amenazas comunes. Los nuevos paradigmas militares dan legitimidad a su actuación al saber actuar de manera terminante y en defensa de los intereses establecidos, es decir, militares bien preparados profesionalmente y adaptables a los cambios pero precisos y motivados en cumplir su misión.

Haciendo una deducción entre los dos paradigmas de seguridad y de la doctrina militar se concluye que el uno va complementado al otro. Si el paradigma de la seguridad ha ampliado sus límites, los cambios en el profesional de la milicia también, partiendo en la organización de los ejércitos, doctrina, estrategias, capacidades de operación, tecnología, medios de comunicación y todo lo que necesita para enfrentarse a sus nuevos campos de operación. Su ampliación de acción además de combatiente es de cooperante, asistente y reconstructor. Lo relacionado a la estructura y mando es otro eje que influye en el nuevo paradigma por los cambios tecnológicos que modifican las capacidades estratégicas, haciendo evolucionar las tácticas, sistemas logísticos y hasta parámetros psicológicos (Moliner, 2012)

No obstante, es indispensable admitir el nuevo paradigma de la seguridad interna como parte substancial de la seguridad integral; y la intervención de las Fuerzas Armadas de igual manera aplicando ya sea un nuevo paradigma o la transformación paradigmática del militar que sea sostenible, compaginativo entre los dos y esenciales para su cabal cumplimiento. Sin embargo, es fundamental conocer los conceptos tanto de seguridad integral como de doctrina militar de manera completa, dominar sus características, sus alcances y limitaciones, la clarificación de su importancia y trascendencia. Esto permitirá poder aceptarlos y percibir que tanto la seguridad como las Fuerzas Armadas han cambiado y deben seguir reemplazando sus tácticas, doctrinas y estrategias para poder operar en los diferentes escenarios que por supuesto también se han transformado.

Para que este paradigma sea completo es importante la educación y la asesoría técnica en materia de Derechos Humanos para y desde las Fuerzas Armadas, cambiar desde el estatocentrismo hacia modelos de seguridad democrática, dando prioridad a las personas, en su entorno familiar, sus derechos civiles así como los derechos militares, cuidado con el medio ambiente y proporcionar todos los medios necesarios para du desarrollo y autorrealización.

Estos cambios evocan a la creación de nuevas leyes, normas y reglamentos que se adapten plenamente a los nuevos paradigmas que además no solo involucran a los relacionados con la seguridad y la doctrina militar, estos envuelven otros como el del conocimiento, la cultura, los derechos y otros. Las leyes deben ser creadas para proteger el accionar del militar en su derecho y obligación de intervenir en la seguridad interna sin temor a ser juzgado, sin ser el caso de un mal comportamiento o abuso de autoridad.

Para el caso de Ecuador, el análisis de los cambios paradigmáticos podría ayudar a comprender la necesidad de la intervención de las Fuerzas Armadas en la seguridad interna. Esta misma necesidad obliga a la preparación de las FF.AA. en el campo propuesto, la formación profesional en primera instancia debe girar en torno al pleno conocimiento de los derechos humanos y al manejo de estos en casos de conflictos, las estrategias de táctica y mando encaminados al enfrentamiento con su propia gente que es diferente al enfoque de guerras externas. Este nuevo tipo de conflictos, unidos a la plena conciencia de la propia naturaleza del militar basados en la responsabilidad de proteger deben conjugarse en una estrategia de defensa usando sus capacidades operacionales para garantizar la seguridad interna como eje de la seguridad integral.

Los expertos en guerras y antiguos protagonista como el General MacArthur explicaba en dos palabras el fracaso en la guerra "demasiado tarde. Demasiado tarde en comprender el propósito mortífero de un enemigo potencial, demasiado tarde en la preparación, demasiado tarde en reunir todas las fuerzas necesarias, demasiado tarde en comprometerse con los amigos" (Gómez, 2012, pág. 125). Esperar comprender y adaptarse a los cambios puede ser demasiado tarde para que la seguridad interna del país se vea involucrada con grupos perturbadores, ya sean delincuentes, terroristas, narcotraficantes, al final el titulo no importa, la inseguridad sí, marca temor, miedo, complejos y retrocesos en el desarrollo. Prepararse tanto interna como externamente es la solución para mejorar la seguridad del país.

Los acontecimientos de finales de 2019 en toda Latinoamérica obligan a reflexionar sobre todos los aspectos de seguridad y la inseguridad progresiva. Koffi Annan secretario de la ONU decía: “¿se puede permitir que existan violaciones masivas y sistemáticas de los derechos humanos, con graves consecuencias humanas sin tomar acción? (citado en Gómez, 2012), exhortando a la responsabilidad de proteger por parte de las Fuerzas Armadas, al no mantener la impunidad o atar su participación bajo leyes propuestas por un Estado incapacitado. Las FF.AA. por su parte garantizan una mayor capacidad de despliegue, mantienen estrategias con capacidades de reacción rápidas y efectivas. 
Todo lo expuesto tanto por el lado de la seguridad como por parte de las FF.AA. no se cumple en su totalidad si no existe un adecuado equipamiento. Enfrentarse a grupos armados y tecnológicamente capacitados en superioridad no garantiza la continuidad del esfuerzo realizado por estabilizar positivamente un conflicto. La misma asimetría de las amenazas implica la necesidad de equipos y armamento de defensa apropiado.

\section{Conclusiones}

La conclusión a este artículo se reduce a la comprensión de los limites extensibles de la seguridad y de la doctrina militar, esto aportaría con la comprensión y aceptación de su empleo en la seguridad interna del país; su capacitación para enfrentamientos internos, la protección legal de su intervención; y por último, el equipamiento apropiado para su participación en la seguridad interna.
Esta conclusión exhorta a la primera hipótesis planteada confirmando la necesidad de las Fuerzas Armadas en ampliar su doctrina para enfrentar las nuevas amenazas multidimensionales.

En esta investigación además se describió la situación en algunos países de Latinoamérica para centrarnos en Ecuador. Toda la región insertada en una situación de conflictos políticos, sociales y delincuenciales viéndose obligados sus gobiernos a la intervención de las Fuerzas Armadas. Esto justifica una vez más la primera hipótesis planteada y confirma la segunda que relaciona los dos paradigmas militar y de la seguridad interna.

Una vez aceptado el nuevo paradigma militar justifica la necesidad de una participación inclusiva e integral, atendiendo las dos seguridades interna y externa, sin dejar de manera secundaria su capacitación y tecnificación, por el mismo hecho de enfrentarse a nuevos escenarios de conflictos globalizados y asimétricos.

\section{REFERENCIAS}

[1] Acosta, F. (2009). ¿Sabes realmente qué es un paradigma? Revista Iberoamericana de Educación, 1-10.

[2] Algora, M. (2012). Orden y Desorden Internacional en el mundo contemporáneo. En V. autores, En una sociedad posheroica: La transformación del paradigma militar (págs. 47-74). Madrid: Ministerio de Defensa.

[3] Álvarez, D., Garzón, J., Tellería, L., \& Paz, M. (2012). El papel de las Fuerzas Armadas en Améica Latina Seguridad interna y democracia. Buenos Aires: CLACSO.

[4] Bellota, L. (2016). Revista Legislativa de Estudios Sociales y de Opinión Pública, 165-187.

[5] Borrero, A. (2003). La actualdiad del pensamiento de Carl von Clausewitz. Revista de Estudios Sociales.

[6] Brandt, W. (1980). Comisión Brandt. Recuperado el 2 de enero de 2020, de https://www. jstor.org/stable/23395590?readnow $=1 \&$ seq $=1$ \#page_scan_tab_ contents

[7] Buzan, B. (1991). People States and Fear: An Agenda for International Security Studies in the Post Cold War Era. Colorado: Lynne Rienner Publishers.

[8] Capponi, R. (2019). Felicidad sólida. Sobre la construcción de una felicidad perdurable. Madrid: Caligrama.

[9] Centro Internacional de Toledo por la Paz CITpax. (2009). Los nuevos paradigmas de la seguridad. Recuperado el 30 de diciembre de 2019, de http://www.toledopax. org/sites/default/files/CITpax IEEE Nuevos paradigmas de la Seguridad_diciembre_2009_0.pdf
[10] Colom, G. (2014). La revolución militar posindustrial. Revistas de Estudios Sociales, 113-127.

[11] Fuerzas Armadas del Ecuador. (mayo de 2014). Revista Fuerzas Armadas del Ecuador(150).

[12] Gómez, M. (2012). ¡A mí la Legión! En V. Autores, En una sociedad posheroica: La transformación del Paradigma Militar (págs. 111-140). Madrid: Ministerio de Defensa.

[13] Grupo de Trabajo en Seguridad Regional (GTSR). (2013). Los militares en la seguridad interna: realidad y desafios para Ecuador. Recuperado el 21 de diciembre de 2019, de https://library.fes.de/pdffiles/bueros/la-seguridad/10063.pdf

[14] Jarrín, O. (11 de agosto de 2011). Nuevo paradigma de seguridad. $E l$ Comercio.

[15] Kuhn, T. (1971). La estructura de las revoluciones científicas. Chicago: Fondo de Cultura Económica.

[16] López, E. (2011). Las Fuerzas Armadas en las calles. Revista de Derechos Humanos, 6-8.

[17] Medina, I. (2015). Apoyo de las FF.AA. a los organismos del Estado ante amenazas y riesgos que atentan a la seguridad integral. Recuperado el 24 de diciembre de 2019, de http://repositorio.espe.edu.ec/xmlui/ handle $/ 21000 / 10292 /$ browse?value= Medina + Jim $\%$ C3\%A9nez\%2C $+\mathrm{Iv} \% \mathrm{C} 3 \% \mathrm{~A} 1 \mathrm{n}+$ Patricio\&type $=$ author

[18] Ministerio de Defensa Nacional. (2006). Libro Blanco del Ecuador. Quito.
[19] Ministerio de Coordinación de Seguridad. (2011). Plan Nacional de Seguridad Integral. Recuperado el 24 de diciembre de 2019, de http:// instrumentosplanificacion.senplades. gob.ec/documents/20182/22941/ PlanNacionaldeSeguridadIntegral 2011.pdf/3dd2fe8a-e2dd-4ab9-87d8$54 \mathrm{eee} 6485 \mathrm{~d} 58$

[20] Ministerio de Defensa. (2018). Política de la Defensa Nacional del Ecuador "Libro Blanco". Quito: Instituto Geográfico Militar.

[21] Ministerio de Defensa Nacional. (2019). Plan Nacional de Seguridad Integral 2019-2030. Quito: Instituto Geográfico Militar.

[22] Moliner, J. (2012). Héroes o villanos. La transformación en las Fuerzas Armadas. En V. autores, En una Sociedad Posheroica: La transformación del Paradigma Militar (págs. 141-170). Madrid: Ministerio de Defensa.

[23] Oliva, J. (2013). La Seguridad Interior en la Policía de Defensa Nacional del Estado Mexicano. En Á. Sarmiento, La Seguridad Nacional Integral de México. Diagnóstico y Propuestas. México: Centro de Estudios Superiores Navales.

[24] Red de Seguridad y Defensa de América Latina. (2002). Ecuador: Libro Blanco de la Defensa Nacional. Quito: https://www.resdal.org/ Archivo/ecu-libro-cap1.htm.

[25] Revilla, M. (2010). América Latina y los movimientos sociales: el presente de la "rebelión del coro". Nueva Sociedad, 51-67. 